\title{
Hypothyroidism - A cause for dilated cardiomyopathy in dogs; four year study (2008-2011)
}

\author{
Satish Kumar Karlapudi ${ }^{1}$, Devarakonda Srikala², D S Tirumala Rao \\ 1. Department of Veterinary Clinical Medicine, College of Veterinary Science, Rajendranagar, \\ Sri Venkateswara Veterinary University, Hyderabad-30, Andhra Pradesh, India. 2. Department of Veterinary \\ Clinical Medicine, College of Veterinary Science, Tirupati-517502, Andhra Pradesh, India. 3. Director of \\ Research, Sri Venkateswara Veterinary University, Hyderabad-30, Andhra Pradesh, India. \\ Corresponding author: K. Satish Kumar, email: dr_petlover@yahoo.co.in \\ Received: 02-06-2012, Accepted: 03-07-2012, Published online: 07-11-2012 \\ doi: $10.5455 /$ vetworld.2012.742-747
}

\begin{abstract}
Aim: The study was carried out to understand the thyroid dysfunction and its association with dilated cardiomyopathy in dogs. Materials and Methods: The study was done at Teaching Veterinary Hospital, Bhoiguda, College of Veterinary Science, Hyderabad for four years, i.e., from 2008 to 2011 . A total of $256 \mathrm{dogs}$ of various breed, age and sex were presented with typical skin and coat abnormalities. Few were also exhibiting signs of low metabolic rate. Skin sample analysis was done to rule out the causes of dermatitis. Thyroid profile was estimated to diagnose hypothyroidism. Selected cases were also subjected for echocardiography to study the association of cardiomyopathy. Based on thyroid profile, hypothyroid dogs were treated with levothyroxine @ 20mcg/kg wt, once daily, orally on empty stomach and dilated cardiomyopathy (DCM) associated patients were additionally supplemented with enalapril @ $0.5 \mathrm{mg} / \mathrm{kg}$, twice daily, orally for 6 months. The hemato biochemical and echocardiographic aspects are discussed.

Results: The classical signs that were recorded in almost all the thyroid dysfunction dogs (231) were bilateral alopecia, rat tail and pigmentation and whereas, dyspnoea at rest, exercise intolerance, obesity, pale mucosae and corneal lipidosis were the significant low metabolic rate signs noticed in 42 dogs. However, syncope and seizures were also recorded in 31 of these hypothyroid dogs. Echocardiographic evaluation revealed significantly $(\mathrm{P}<0.01)$ increased LVEDd and LVEDs along with decreased IVS and LVPW both at systole and diastole among 33 dogs. Normocytic, normochromic and non-regenerative anemia and significantly $(\mathrm{P}<0.05)$ low T3, fT4, tT4 along with elevated serum cholesterol, triglycerides, TSH, CKMB, LDH and ALP were the hemato - biochemical findings among these dogs. After the initiation of therapy, improvement in clinical signs was noticed from day 7 and complete clinical recovery by the end of therapy. However, a non significant improvement was recorded with respect to left ventricle dimensions.

Conclusions: From the present study it may be concluded that thyroid dysfunction is presented by manifestations of two different systems, i.e., skin and coat and metabolic abnormalities. Echocardiography is the more sensitive diagnostic aid to ascertain the involvement of cardiovascular system and to assess the therapeutic response. Further, supplementation of ACE inhibitors along with the specific thyroid supplements helps to resolve the disorder.
\end{abstract}

Key words: dilated cardiomyopathy, dogs, enalapril, hypothyroidism, levothyroxine

Abbreviations: DCM-Dilated cardiomyopathy, T4-Thyroxine, T3-Tri-iodothyronine, tT4-Total thyroxine, fT4-Free thyroxine, TSH-Thyroid stimulating hormone, LVEDd-Left ventricle end diameter at diastole, LVEDs-Left ventricle end diameter at systole , IVSd-Inter ventricular septum at diastole, IVSs-Inter ventricular septum at systole, LVPWd-Left ventricle free wall at diastole, LVPWs-Left ventricle free wall at systole, EF-Ejection fraction, FS-Fractional shortening, ACEi-Angiotensin converting enzyme inhibitors

\section{To cite thisarticle:}

Karlapudi SK, Srikala D, Rao DST (2012) Hypothyroidism - A Cause for dilated cardiomyopathy in dogs; four year study (2008-2011), Vet World, 5(12): 742-747, doi: 10.5455/vetworld.2012.742-747

\section{Introduction}

Hypothyroidism results in decreased production of thyroid hormones, thyroxine $\left(\mathrm{T}_{4}\right)$ and $3,5,3$ triiodothyronine $\left(\mathrm{T}_{3}\right)$ from the thyroid gland. Naturally occurring hypothyroidism is a common disease in dogs but rare in cats [1]. Thyroid hormones have positive inotropic and chronotropic effects on the heart and have catabolic effects on muscle and adipose tissue, stimulate erythropoiesis and regulate both cholesterol synthesis and degradation. Thyroid dysfunction is characterised by slow, lazy behaviour, bilateral alopecia, dry skin, obesity, lethargy and respiratory distress. Uncommonly, cardiovascular abnormalities are also associated with hypothyroidism. Further, cardiac changes that develop in dogs with hypothyroidism are important because of the 
high incidence of hypothyroidism and heart disease in some breeds [2]. Any alteration in cardiac function induced by hypothyroidism could result in worsening of pre-existing disease [3]. Interest in the role of thyroid hormones on cardiac failure has increased in recent years. The driving considerations can be summarized as follows: (a) the known effects of thyroxine on contractile and relaxation properties of the heart; (b) experimental findings offering strong support for the hypothesis that thyroid hormone signaling is critical in preserving cardiac structure and performance under normal conditions and after cardiac injury; and (c) evidence that thyroid dysfunction is strongly associated with a worsening prognosis in cardiac patients in general and in cardiac failure patients in particular $[4,5]$. Diastolic function and systolic function are clearly influenced by various thyroid hormones [6]. Ventricular contractile function is also influenced by changes in hemodynamic conditions secondary to thyroxine effects on peripheral vascular tone [7]. The homeostasis of these hormones preserves positive ventricular-arterial coupling, leading to a favorable balance for cardiac work. Other studies suggest reduced cardiac tissue triiodothyronine levels after myocardial infarction (MI) or with development of hypertension by upregulating type 3 deiodinase (D3), which leads to deactivation of triiodothyronine and thyroxine [8].

Keeping this hypothesis in view the present study was carried out to evaluate the association of cardiomyopathy with thyroid dysfunction in dogs.

\section{Materials and Methods}

A total of 256 dogs of various breed, age and sex that were presented from 2008 to 2011 to the Veterinary Hospital, Bhoiguda, College of Veterinary Science, Hyderabad, with the classical signs of skin and coat and metabolic abnormalities were considered for the study. Almost all of these dogs were treated for dermatitis by local Veterinarian, but the condition was reported to be recurring when therapy discontinued. The owners further reported that, there was no improvement with respect to the signs associated with low metabolic rate in few of the cases. Thorough clinical examination was followed by blood and serum analysis. Further, the hypothyroid patients presenting signs associated with reduced metabolic rate (cyanotic tongue, dyspnoea at rest, lethargy and exercise intolerance) were suspected for cardiac involvement and were subjected for electrocardiography, thoracic radiography and 2-d echocardiography. Echocardiogram was carried out using a microconvex array c5-2
R13 cardiac probe placed on right precardium, with dogs positioned in right lateral recumbancy. Access to the right side of the thorax was facilitated by use of a table with a special cut-out to allow the transducer to be directed upward towards the site of maximal cardiac pulsation. Transducer is located parasternally between right third and sixth intercostal spaces between sternum and costochondral junction. M-mode recordings were taken at the high papillary level. However, echocardiogram measurements were also recorded in apparently healthy dogs to establish normal values. Based on these findings 33 hypothyroid patients diagnosed for associated dilated cardiomyopathy were selected and were treated with levothyroxine @ 20 $\mathrm{mcg} / \mathrm{kg}$, once daily on empty stomach and enalapril @ $0.5 \mathrm{mg} / \mathrm{kg}$ once daily for $6 \mathrm{months}$. However, levothyroxine was also supplemented for the remaining hypothyroid cases for 6 months. The blood and sera samples were collected as per the animal ethics and the therapy was also carried out as per the ethical treatment protocol.

\section{Results}

Almost all the hypothyroid dogs revealed similar clinical manifestations viz., bilateral symmetrical alopecia, rat tail appearance, pigmentation, bald thigh, weakness, cold intolerance and lethargy. Whereas, dyspnoea at rest, exercise intolerance, corneal lipidosis and cyanotic tongue were recorded in 41 cases and seizures and syncope among 31 . The mean weight and age was $45 \mathrm{~kg}$ and $8 \mathrm{yrs}$, respectively. Hematologically, non-regenerative anemia and biochemically, significant increase $(\mathrm{P}<0.05)$ in serum cholesterol, triglycerides, alkaline phosphatase (ALP) with a significantly decreased $(\mathrm{P}<0.05)$ levels of tri-iodothyronine $(\mathrm{T} 3)$, total thyroxine (tT4), free thyroxine (fT4) and increased thyroid stimulating hormone (TSH) were recorded in 231/256 dogs, along with a significantly increased $(\mathrm{P}<0.05)$ creatine kinase MB (CKMB) and lactate dehydrogenase (LDH) in 33 hypothyroid dogs in comparison with apparently healthy dogs (Table-1). These dogs with abnormal cardiac markers were suspected for cardiomyopathy and subjected for further detailed investigations. Low voltage $\mathrm{QRS}$ complexes and ventricular premature complexes were the significant electro cardiographic (ECG) findings and whereas, cardiomegaly was the radiographic abnormality. Bmode echocardiography revealed round greatly enlarged globose shaped heart (fig. 1). A significantly $(\mathrm{P}<0.01)$ increased left ventricle end diameter (LVED) along with significantly $(\mathrm{P}<0.05)$ decreased interventricular septum (IVS) and left ventricle free wall (LVWP) dimensions both at diastole (d) and systole (s) 


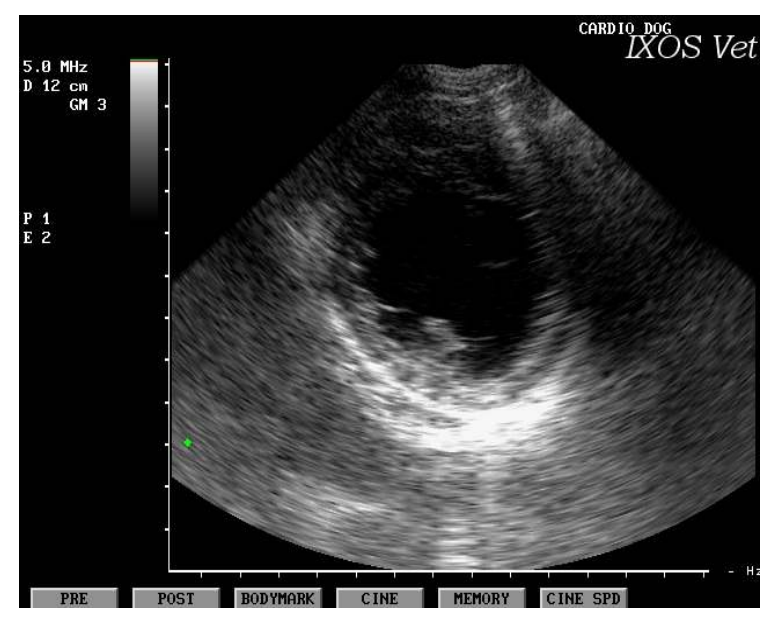

Fig.1. B-mode echocardiogram showing round globose left ventricle (day 0 )

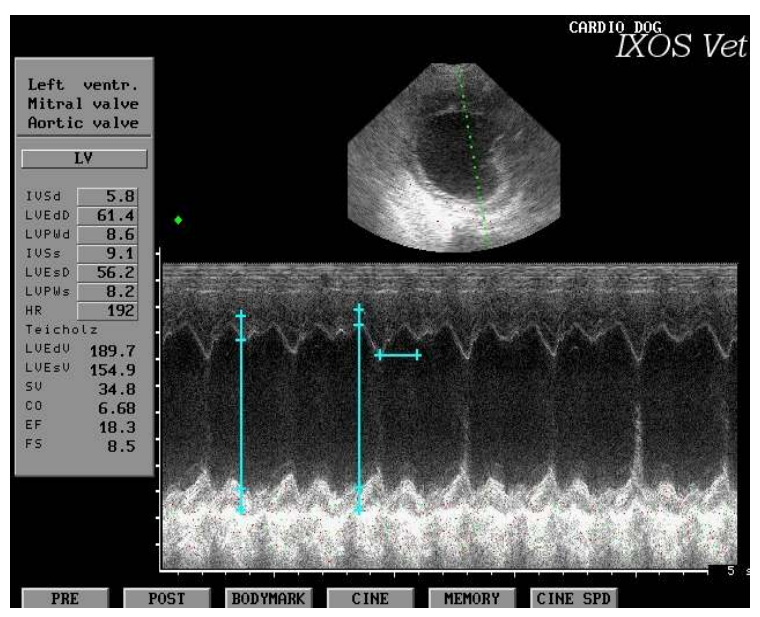

Fig.2. M-mode echocardiogram showing abnormal left ventricle dimensions and indices (day 0 )

Table.1 Serum profile hypothyroid associated Cardiomyopathy dogs

\begin{tabular}{|c|c|c|c|c|c|}
\hline SI. No. & Parameter & Healthy dogs & $\begin{array}{l}\text { DCM dogs } \\
\text { day } 0\end{array}$ & Day 90 & day 180 \\
\hline 1. & Cholesterol (mmol/L) & $4.36 \pm 0.20$ & $14.12^{*} \pm 0.22$ & $11.80 \pm 0.44$ & $6.00 * \pm 0.22$ \\
\hline 2. & Triglycerides (mmol/L) & $1.40 \pm 0.84$ & $6.10^{\star} \pm 0.24$ & $5.20 \pm 0.48$ & $2.32^{*} \pm 0.04$ \\
\hline 4. & fT4 (ng/dl) & $2.72 \pm 0.50$ & $0.70^{\star} \pm 0.08$ & $1.14 \pm 0.08$ & $2.12^{*} \pm 0.20$ \\
\hline 5. & T3 (ng/dl) & $69.20 \pm 0.28$ & $31.50^{\star} \pm 0.76$ & $43.88 \pm 0.70$ & $61.08^{\star \star} \pm 1.24$ \\
\hline 6. & TSH (ng/ml) & $2.12 \pm 0.22$ & $26.00^{*} \pm 0.80$ & $19.00 \pm 0.74$ & $4.88^{* *} \pm 0.22$ \\
\hline 9. & CKMB (U/L) & $24.50 \pm 0.84$ & $60.18^{* *} \pm 1.50$ & $55.20 \pm 0.20$ & $24.2^{* *} \pm 1.16$ \\
\hline 10. & $\mathrm{LDH}(\mathrm{U} / \mathrm{L})$ & $286.46 \pm 1.80$ & $696.50^{\star \star} \pm 2.22$ & $622.18 \pm 1.20$ & $318.44^{\star *} \pm 1.98$ \\
\hline
\end{tabular}

* significant at 5 per cent $(\mathrm{P}<0.05)$; ** significant at 1 per cent $(\mathrm{P}<0.01)$

and Ejection fraction (EF) and Fractional Shortening (FS) were the significant m-mode echocardiographic measurements. Thus, confirming associated dilated cardiomyopathy among 33 hypothyroid dogs of which German shepherd (14) followed by Labrador retriever (09) were commonly affected breeds (fig. 2; table. 2).

Improvement in skin and coat associated signs were noticed from day 7 followed by signs associated with low metabolic rate and complete clinical recovery with alleviation of all the signs by the end of therapy. A non- significant improvement in haematological, radiological and electrogardiographic findings were noticed by day 180 . Whereas, significant improvement in biochemical and thyroid profile was noticed from day 90 and reached to near normal by the end of the therapy (Table-1). With respect to left ventricle dimensions on m-mode echocardiography, a nonsignificant alteration was noticed on day 90 , but a significant $(\mathrm{P}<0.05)$ improvement was recorded with respect to LVEdD, LVEsD and EF and FS by the end of therapy (day 180). However, a non- significant increase in IVSd, IVSs, LVPWd and LVPWs were also recorded (fig. $3 \& 4$; table. 2 ).

\section{Discussion}

Hypothyroidism is the common endocrinological disease suspected mostly in canine suffering from non-pruritic alopecia, bald thigh syndrome, rat tail appearance with brittle, dry and change in coat colour and scaling of skin [2]. In the present study common manifestations include abnormalities associated with skin and coat followed by those related to decreased metabolic rate. Dermatological changes occur in 60 to 80 per cent of hypothyroid dogs [3]. The degree of obesity is usually moderate, although obesity is the presenting complaint in dogs of decreased thyroid function [9]. These abnormalities could be attributed to general metabolic derangements that occur in impaired thyroid function or may be an indication of a neuropathy or myopathy [10]. The 
Hypothyroidism - A cause for dilated cardiomyopathy in dogs; four year study (2008-2011)

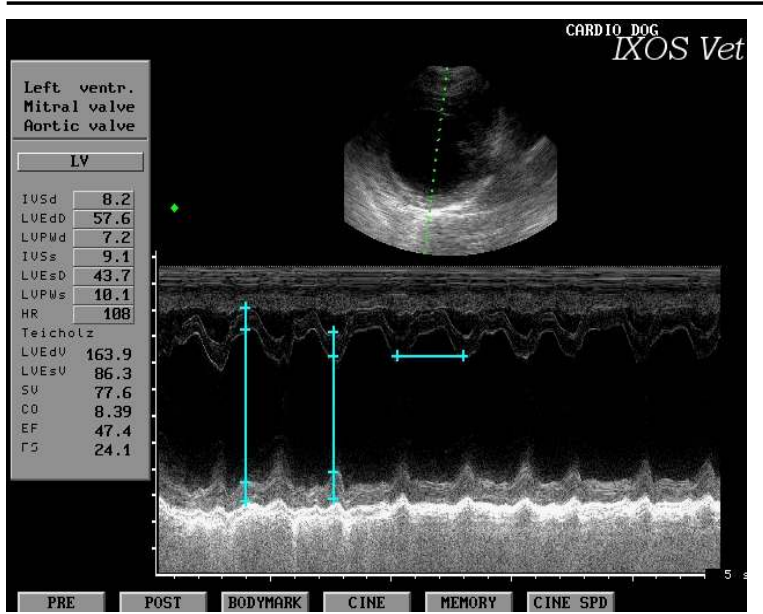

Fig.3. M-mode echocardiogram showing partially improved left ventricle dimensions and indices (day 180)

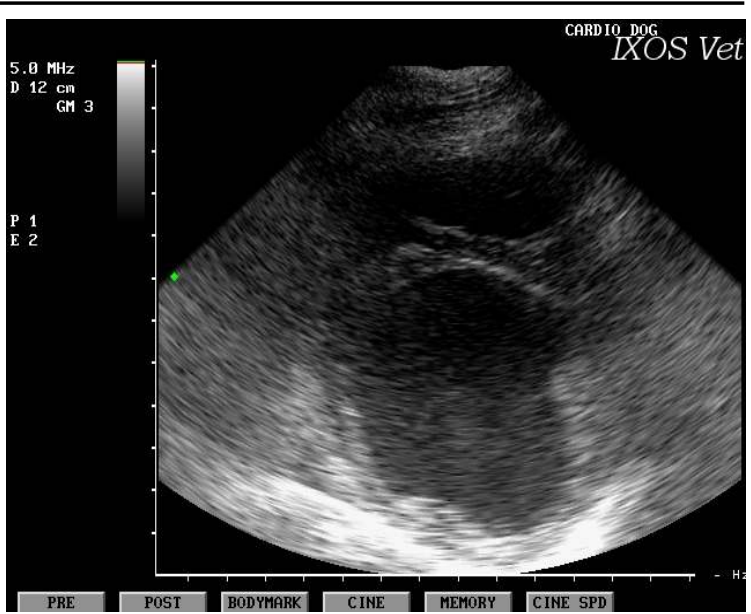

Fig.4. B-mode echocardiogram showing partially contracting left ventricle (day 180)

Table.2. Left Ventricle dimensions and Indices of DCM dogs

\begin{tabular}{|c|c|c|c|c|c|}
\hline SI. No. & Parameter & Healthy dogs & $\begin{array}{l}\text { DCM dogs } \\
\text { day } 0\end{array}$ & Day 90 & day 180 \\
\hline 1. & LVEdD (mm) & $38.72 \pm 0.22$ & $64.52^{*} \pm 1.82$ & $61.10 \pm 0.96$ & $55.30^{* *} \pm 1.20$ \\
\hline 2. & LVEsD (mm) & $29.68 \pm 0.44$ & $55.20^{*} \pm 1.50$ & $50.10 \pm 0.24$ & $43.70 * \pm 1.84$ \\
\hline 4. & LVPWs (mm) & $9.48 \pm 0.80$ & $6.00^{*} \pm 0.52$ & $6.80 \pm 0.20$ & $7.98^{*} \pm 0.28$ \\
\hline 5. & IVSd (mm) & $9.00 \pm 0.46$ & $5.10^{*} \pm 0.04$ & $6.00 \pm 0.26$ & $7.90 * \pm 0.22$ \\
\hline 6. & IVSs (mm) & $10.10 \pm 0.06$ & $6.00^{*} \pm 0.32$ & $7.12 \pm 0.08$ & $8.88^{\star} \pm 0.42$ \\
\hline
\end{tabular}

* significant at 5 per cent $(P<0.05) ; * *$ significant at 1 per cent $(P<0.01)$

signs associated with low metabolic rate viz., general weakness, exercise intolerance, lethargy and listlessness observed in hypothyroidism might be attributed to poor conduction of electrical impulses in hypothyroid patients [11]. Ocular changes are not common in hypothyroidism but the high levels of blood cholesterol and circulating fat can sometimes leads to corneal dystrophy. This finding is usually represented as a small white spot on the eye surface $[12,13]$. Nonregenerative anaemia, hypercholesterolemia and hypertriglyceridemia, with mild increase in alkaline phosphatase, alanine amino transferase and creatine kinase activities were the common hemato-biochemical alterations noticed in the present study were in agreement with the previous authors $[14,15]$.

The significant ECG findings among hypothyroid patients include bradycardia, low voltage QRS complexes and ventricular premature complexes [16]. Experimental studies of hypothyroid dogs have confirmed that atrioventricular conduction time, functional refractory period of the atrioventricular node and duration of ventricular action potential are prolonged. Many of these abnormalities reverse with thyroid hormone supplementation and there appears to be a direct correlation between the electrocardiographical changes and the severity of thyroid deficiency [17].

Significantly decreased left ventricle dimensions and indices with poor left ventricle function was noticed among the DCM affected hypothyroid dogs. A significant increase in LVEDd and LVEDs and decrease in IVSd, IVSs, LVPWd, LVPWs and fractional shortening and ejection fraction were recorded in clinically affected hypothyroid human beings and dogs $[3,18]$. Whereas, decreased left ventricle end diameter during diastole, left ventricle posterior wall during diastole and systole and fractional shortening as compared with baseline values were noticed in experimentally induced hypothyroid dogs [19].

The difference in these dimensions between experimentally induced and clinically affected hypothyroid dogs could be due to a difference in the severity or duration of hypothyroidism in the experimental and 
clinically affected dogs. In the present investigation the DCM dogs were reported to be showing clinical signs suggestive of hypothyroidism for a long period before diagnosis.

Infiltration of the myocardium with mucopolysacharides or impaired coronary arterial vasodilatory ability, possibly secondary to increased alphaadrenergic activity or atherosclerosis, might account for prolonged impairment of cardiac function [11]. In addition alterations in peripheral circulation may return to normal slowly. Some markers of hypothyroidism at tissue level change slowly following levothyroxine supplementation and normalisation of many clinical abnormalities often requires months of thyroid hormone supplementation [20]. Because a complete turnover of protein occurs in the heart approximately every three weeks [21], the 6 months period of levothyroxine administration in the present study seems adequate to allow resolution of most of the cardiac changes.

Following therapy with angiotensin converting enzyme inhibitors (ACEi) and levothyroxine, improvement in clinical signs were recorded. Although non-significant, but improvement in $\mathrm{P}$ and $\mathrm{R}$ wave amplitudes with normal heart rate was also noticed. With respect to echocardiographic abnormalities noticed in DCM associated hypothyroid dogs, significant decrease in LVEDd and LVEDs with an increase in EF and FS was noticed. In the myocardium, thyroid hormone controls myosin enzyme production, sarcolemma calcium ATPase activity, sodium-potassium ATPase activity, calcium channel activity and B-and possibly alpha-adrenergic receptor number and activity. These changes act along with changes in the peripheral circulation including decreased vascular resistance volume and increased systemic vascular resistance, to decrease cardiac output. The relative importance of each factor is unclear, but reversible cardiomyopathy has been documented in hypothyroid people. The cardiac changes that develop in dogs with hypothyroidism are important because of the high incidence of hypothyroidism and heart disease [22]. Any alteration in cardiac function induced by hypothyroidism could result in worsening of pre-existing cardiac complaint, leading to death. Supplementation of ionotropes, ACE inhibitors in addition to the thyroxine have an added advantage in hypothyroid canine patients that help a faster and complete recovery [2]. Though cardiac function usually is adequate to meet tissue needs in patients without underlying cardiovascular disease, pre-existing myocardial dysfunction or systemic arterial hypertension in a hypothyroid individual could compound the effects of hypothyroidism and result in heart failure. Indeed, some hypothyroid people manifest signs of congestive heart failure even in the absence of other underlying cardiac disease [23].

\section{Conclusions}

From the present findings, it may be concluded that thyroid dysfunction not only presents the signs of skin and coat abnormalities, but also exhibits the signs associated with low metabolic rate. A thorough clinical evaluation and clinical chemistry is required to suspect the involvement of cardiovascular system. However, 2-d chocardiography is highly specific and sensitive diagnostic aid to ascertain the involvement of cardiovascular system and to assess the therapeutic response. Once diagnosed, supplementation of ACE inhibitors along with the specific thyroid supplements helps to resolve the disorder.

\section{Author's contribution}

SKK and DS carried out the study under the guidance of DSTR. All authors read and approved the final manuscript.

\section{Acknowledgements}

The authors are thankful to the Sri Venkateswara Veterinary University for providing the facilities at the Veterinary Hospital Bhoiguda, for carrying out the present research.

\section{Competing interests}

Authors declare that they have no competing interests.

\section{References}

1. Rand, JS; Levin, J; Best, SJ and Parker, W (1993). Spontaneous adult onset of hypothyroidism in a cat. Journal of Veterinary Internal Medicine . 7: 272-276.

2. Julie, AF and John, P (2009). Hoover Improvement in myocardial dysfunction in a hypothyroid dog Can Vet J. 50(8): 828-834.

3. Fialkovicova, M; Gaalova, M and Kozak, M (2008). Hypothyroidism and dilative cardiomyopathy in a Great Dane dog. Indian Vet. J. 85: 719-722.

4. Anthony, MG and Giorgio, I (2010). Thyroid Replacement Therapy and Heart Failure. Circulation. 122:385-393.

5. Pol, CJ; Muller, A and Simonides, WS (2010). Cardiomyocyte-specific inactivation of thyroid hormone in pathologic ventricular hypertrophy: an adaptative response or part of the problem? Heart Fail Rev. 15; 133-142.

6. Dillmann, W (2010). Cardiac hypertrophy and 
thyroid hormone signaling. Heart Fail Rev. 15; 125-132.

7. Coceani, M; Iervasi, G; Pingitore, A; Carpeggiani, C and L'Abbate, A (2009). Thyroid hormone and coronary artery disease: from clinical correlations to prognostic implications. Clin Cardiol. 32; 380-385.

8. Passino, C; Pingitore, A; Landi, P; Fontana, M; Zyw, L; Clerico, A; Emdin, M and Iervasi, G (2009). Prognostic value of combined measurement of brain natriuretic peptide and triiodothyronine in heart failure. J Card Fail. 15: 35-40.

9. Panciera, DL (2000). Cardiovascular complications of thyroid disease. In: Bonagura JD, editor. Kirk's Current Veterinary Therapy XIII Small Animal Practice. Philadelphia: WB Saunders; pp. 716-719.

10. Celona, B; Martin, MWS and Stafford JMJ (2009). Canine dilated cardiomyopathy: a retrospective study of signalment, presentation and clinical findings in 369 cases. J. Small Anim. Prac. 50; 23-29.

11. Mayr, A (2007) Generalized Malassezia-dermatitis in a German Shepherd dog with hypothyroidism - a case report. Wiener Tierarztliche Monatsschrift. 94:169-174.

12. Klein, I (2003). Thyroid hormone and cardiac contractility. Am J Cardiol. 91:1331-1332.

13. Durieux, P; Rigaudiere, F; LeGargasson, JF and Rosolen, SG (2008). ERG findings in three hypothyroid adult dogs with and without levothyroxine treatment Vet. Ophthal. 11: 406-411.

14. Williams, DL; Pierce, V; Mellor, P and Heath, MF (2007). Reduced tear production in three canine endocrinopathies. J. Small Anim. Prac. 48: 252-256.

15. Andronic, V; Suvei, I; Andronie, I and Condur, D (2008). Hematological and biochemical modifications in some canine dermatopathies with diverse etiology. Revista Romana de Medicina Veterinara. 18: 229-236.

16. Rossmeisl, JH; Jr. Duncan, RB; Inzana, KD; Panciera, DL and Shelton, GD (2009). Longitudinal study of the effects of chronic hypothyroidism on skeletal muscle in dogs. Am. J. Vet. Res. 70: 879-889.

17. Stephan, I; Nolte, I and Hoppen, HO (2003). Influence of hypothyroidism on cardiac function of dogs. Deutsche Tierarztliche Wochenschrift. 110(6): 231239.

18. Gerritsen, RJ; van den Brom, WE and Stokhof, AA (1996). Relationship between atrial fibrillation and primary hypothyroidism in the dog. Vet $Q .18: 49-51$.

19. Phillips, DE and Harkin, KR (2003). Hypothyroidism and myocardial failure in two Great Danes. JAm Anim Hosp Assoc. 39:133-137.

20. Gaalova, M; Fialkovicova, M; Kozak, $\mathbf{M}$ and Mateova, S (2008). Cardiovascular system abnormalities in a dog with primary hypothyroidism. Medycyna Weterynaryjna. 64: 156-160.

21. Graham, PA; Refsal, KR and Nachreiner, RF (2007). Etiopathologic findings of canine hypothyroidism. (The thyroid.) Veterinary Clinics of North America, Small Animal Practice. 37(4): 617-631.

22. Henderson, KK; Danzi, S; Paul, JT; Leya, G; Klein, I and Samarel, AM (2009). Physiological replacement of T3 improves left ventricular function in an animal model of myocardial infarction-induced congestive heart failure. Circ Heart Fail. 2; 243-252.

23. Julie, AF and John, PH (2009). Improvement in myocardial dysfunction in a hypothyroid dog. Can Vet J. 50(8): 828-834. 